\title{
Wavevector selective surface
}

\author{
V. A. Fedotov ${ }^{1,2, *}$, J. Wallauer ${ }^{3}$, M. Walther ${ }^{3}$, N. Papasimakis ${ }^{1,2}$, N. I. Zheludev ${ }^{1,2,4}$ \\ ${ }^{1}$ Optoelectronics Research Centre, University of Southampton, SO17 1BJ, UK \\ ${ }^{2}$ Centre for Photonic Metamaterials, University of Southampton, SO17 1BJ, UK \\ ${ }^{3}$ Department of Molecular and Optical Physics, University of Freiburg, D-79104 Freiburg, Germany \\ ${ }^{4}$ Centre for Disruptive Photonics Technologies, Nanyang Technological University, 637371 Singapore \\ *vaf@orc.soton.ac.uk
}

\begin{abstract}
Frequency selective surfaces are very well known and have been investigated in detail. Here we for the first time introduce the concept of a wavevector selective surface and demonstrate it experimentally.

OCIS codes: 160.3918 Metamaterials; 070.6110 Spatial filtering
\end{abstract}

\section{Introduction}

We report for the first time a new class of planar metamaterials that act as a wavevector-selective surfaces (WSS): they discriminates incident waves by their wavevector and are transparent in a narrow range of light propagation directions, thus operating as a tunnel vision filters. Such filtering functionality results from the collective in-phase excitations of strongly interacting metamolecules. It does not rely on gradient structuring of the metamaterials and hence applies to spherical wavefronts (and their superpositions) of any curvature and any point of origin. The wavevector selective surface can be exploited for enhancing observational instruments, long-distance free-space communication lines and for cloaking applications.

\section{Simulations}

We demonstrated the effect of wavevector filtering computationally, by simulating the propagation of spherical wavefronts through a finite-sized coherent metamaterial. It was modeled by a planar array of $14 \mathrm{x} 14$ identical subwavelength metallic resonators shaped as an asymmetrically split ring (ASR). Such a metasurface was designed to operate with no diffraction in the lower part of the terahertz spectrum, at frequencies below $0.3 \mathrm{THz}$, and in the case of plane-wave illumination its spectrum showed a narrow EIT-like transmission resonance centered at around 0.165 THz.
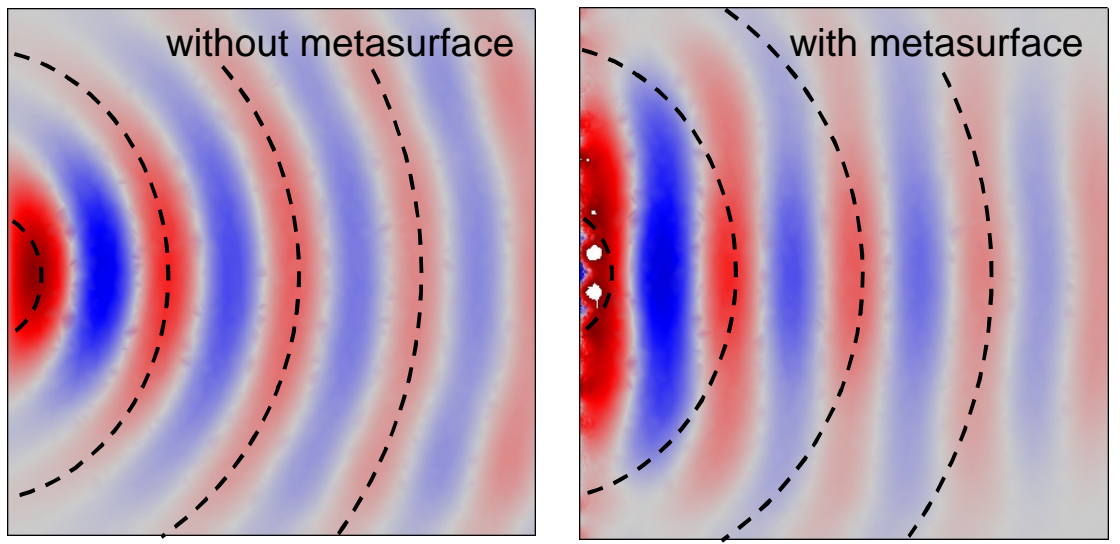

Fig. 1. Planar metamaterial as a wavevector selective surface. Propagation of spherical waves simulated with and without the metamaterial in place. The waves are incident from the left. The dashed traces indicate the curvature of the wavefronts produced by an ideal point source. 
The resonance is induced by the component of electric field parallel to the split of the rings and is attributed to antisymmetric currents resonantly excited in the opposite arcs. Such a current mode, when induced by a normally incident plane wave, does not radiate into the far field, which makes the mode isolated or trapped (trapped-mode resonance) promoting strong inter-metamolecular interactions through surface-bound magneto-inductive waves it creates. It is the strong inter-metamolecular coupling that enables wavevector filtering in planar metamaterials.

Figures 1 illustrates this effect at $0.163 \mathrm{THz}$ for the case of propagating spherical waves, which are produced by an electromagnetic point source placed close to the metasurface, at the distance equal to just one period of the ASRarray. Here we compare propagation of the spherical waves simulated with and without the metasurface in place. Evidently, the waves propagating through the empty space remain spherical, while their interaction with the metasurface makes the transmitted wavefronts nearly planar. We have also found that in the former case the angular spectrum of the admitted wavevectors appeared isotropic, while in the latter case the spectrum converged along the normal to the metasurface, thus signifying the regime of wavevector filtering.

\section{Experiment}

Our findings have been fully confirmed experimentally. For this we fabricated a WSS-metamaterial sample as the exact copy of the modeled ASR-array. The spherical waves were produced by illuminating a $1750 \mu \mathrm{m}$ large pinhole placed $500 \mu \mathrm{m}$ away from the sample. The wavefronts were mapped using a state-of-the-art THz-field imaging technique, which enabled accurate spatial mapping of electric-field component of the transmitted waves with the frequency resolution of $0.03 \mathrm{THz}$. The obtained wavefront patterns, as well as their spatial-frequency spectra, showed a very good agreement with the results of our simulations demonstrating robustness of the predicted effect.

\section{Summary}

We report on, what we believe, the next significant development in the domain of controlling and manipulating light with metasurfaces, the wavevector filtering. Several important applications of the new functionality will be discussed, including enhancement of observational instruments, long-distance free-space communications in strongly scattering environment, and new-principle cloaking.

\section{References}

[1] N. Yu, P. Genevet, M. A. Kats et al., "Light propagation with phase discontinuities: generalized laws of reflection and refraction", Science 334, 333 - 336 (2011).

[2] A. S. Schwanecke, V. A. Fedotov, V. V. Khardikov et al., "Nanostructured metal film with asymmetric optical transmission", Nano Lett. 8, $2940-2944$ (2008).

[3] E. Plum, X.-X. Liu, V. A. Fedotov et al., "Metamaterials: optical activity without chirality”, Phys. Rev. Lett. 102, 113902 (2009). 